\title{
Einzel lens chopper and behavior of the chopped beam in the KEK digital accelerator
}

\author{
K. W. Leo, ${ }^{1,2}$ T. Adachi, ${ }^{1,2}$ T. Arai, ${ }^{2}$ and K. Takayama ${ }^{1,2,3}$ \\ ${ }^{1}$ The Graduate University for Advanced Studies (KEK), Tsukuba, Ibaraki 305-0801, Japan \\ ${ }^{2}$ High Energy Accelerator Research Organization (KEK), Tsukuba, Ibaraki 305-0801, Japan \\ ${ }^{3}$ Tokyo Institute of Technology, Nagatsuda, Kanagawa 152-8550, Japan
}

(Received 9 August 2012; published 17 April 2013)

\begin{abstract}
The KEK digital accelerator (KEK-DA), which is a small-scale rapid-cycle induction synchrotron (IS), has commenced operation. A permanent magnet $x$-band electron cyclotron resonance ion source serves as the KEK-DA ion source and delivers various ions. A new Einzel lens beam chopper has been developed to provide the necessary pulse width of a few microseconds. The chopper is implemented by applying a rectangular pulse voltage generated by a solid-state Marx generator to the middle electrode of the Einzel lens. Momentum modulation at the bunch head and tail resulting from chopping in longitudinal direction has been observed. This intrinsic property of the chopped pulse has been clearly observed in a long drift (a free run in the circular ring) in KEK-DA.
\end{abstract}

DOI: 10.1103/PhysRevSTAB.16.043502

PACS numbers: $29.20 . \mathrm{dk}$

\section{INTRODUCTION}

\section{A. KEK-DA}

The KEK digital accelerator (KEK-DA) is a small-scale induction synchrotron (IS) that was built as part of the renovation of the former KEK $500 \mathrm{MeV}$ booster ring of the rapid-cycle proton synchrotron [1]. In KEK-DA, an induction cell is employed as an acceleration device instead of a conventional RF cavity [1-4]. The concept of IS was demonstrated experimentally in 2006 [2] by using the KEK $12 \mathrm{GeV}$ proton synchrotron. Although in principle KEK-DA is an injector-free accelerator, certain injection energy is nevertheless required to mitigate the transverse space-charge effect and closed-orbit distortions caused by remnant fields in the ring. For these reasons, the electron cyclotron resonance ion source (ECRIS) is embedded in a high-voltage platform (HVP). Figure 1 shows the KEK-DA complex, where an ion beam produced in ECRIS [5] is extracted with a voltage of $10 \mathrm{kV}$ and accelerated with a post-acceleration column with a voltage of $190 \mathrm{kV}$. The ion beam $\left(\mathrm{He}^{1+}\right.$ was utilized) propagates through the lowenergy beam transport line (LEBT), after which it is injected into the KEK-DA ring with an electrostatic (ES) kicker [1]. Since we use a single-turn injection method, the ES kicker must be turned off within the revolution time of the ion beam after injection. Therefore, the required pulse length should be shorter than the revolution time of the ion beam, or $\sim 12 \mu \mathrm{s}$ for $\mathrm{He}^{1+}$. The pulse width of the beam extracted from ECRIS operated in the pulse mode is of the order of a few milliseconds due to the intrinsic nature of plasma buildup in ECRIS. Thus, a beam chopper must be

Published by the American Physical Society under the terms of the Creative Commons Attribution 3.0 License. Further distribution of this work must maintain attribution to the author(s) and the published article's title, journal citation, and DOI. employed before the injection. Various types of beam choppers are available for this purpose.

\section{B. KEK-DA ECRIS}

Figure 2 shows a schematic view of KEK-DA ECRIS. It is an $x$-band ECRIS of the permanent magnet type, and its details are described in [5,6]. ECRIS is operated in the pulse mode with a repetition rate of $10 \mathrm{~Hz}$, which is the same as the repetition rate of KEK-DA. The pulse mode operation does not require water cooling, which allows us to install ECRIS easily on the HVP. Owing to the fundamental ionization process in ECRIS, it requires a few hundred microseconds for ions with highly charged states to build up, and a few milliseconds to achieve a stable and saturated condition, as shown in Fig. 3. This time interval is

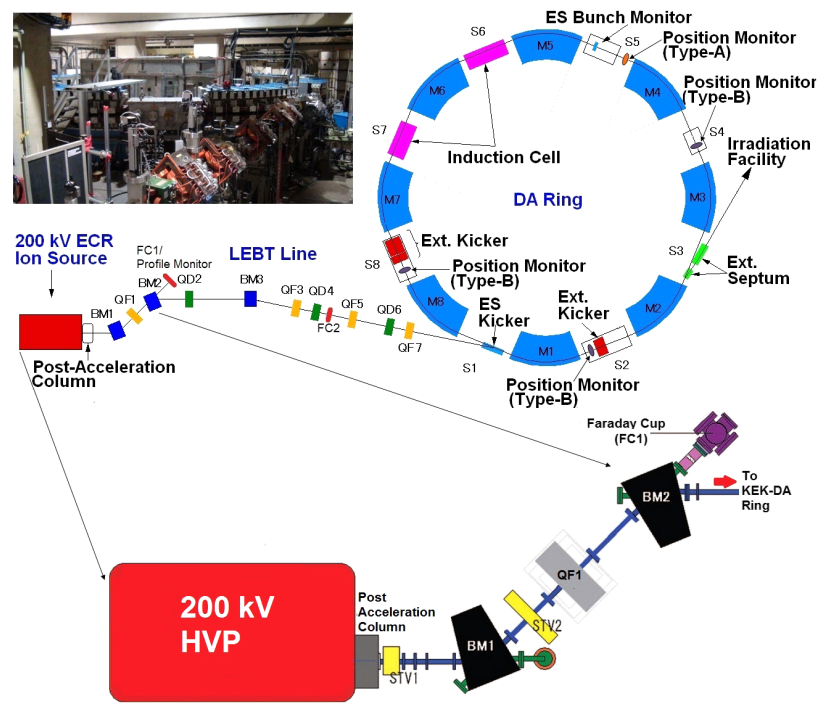

FIG. 1. Layout of the KEK-DA complex and experimental setup, where FC1 is a Faraday cup for ion beam monitoring. 


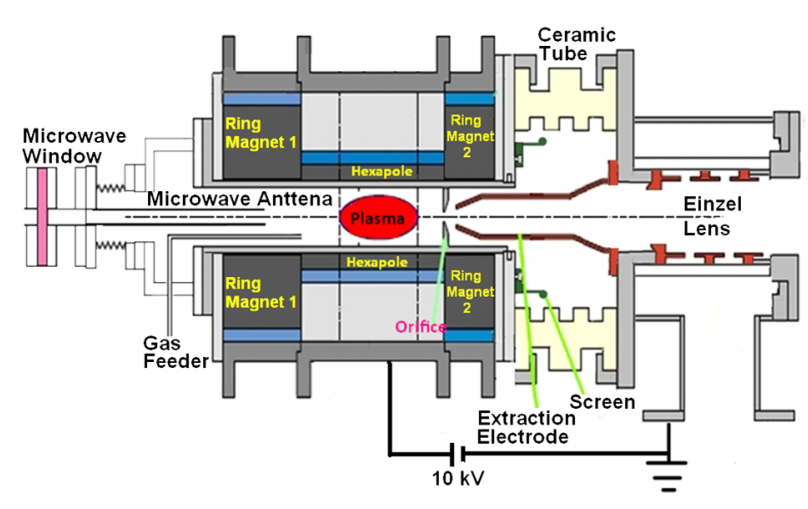

FIG. 2. Schematic view of KEK-DA ECRIS.
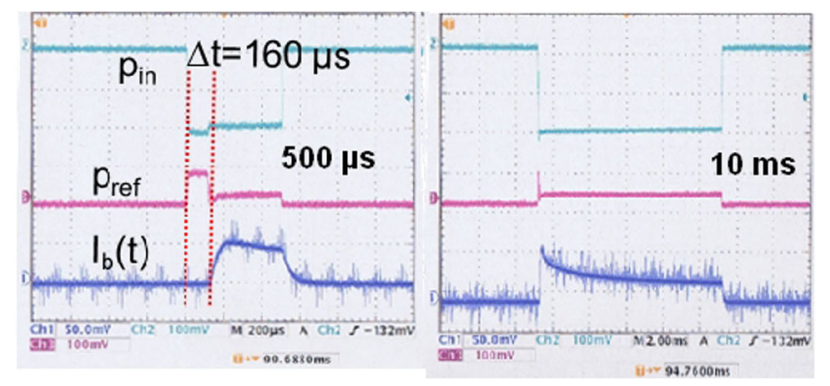

FIG. 3. Typical plasma buildup time with various durations. The green line represents the injected microwave power, the pink line denotes the reflected microwave power, and the blue line represents the ion beam current.

1000 times longer than the required pulse length of $\sim 10 \mu \mathrm{s}$ in KEK-DA, where the so-called single-turn injection scheme has been employed. Thus, 1/1000 of the ion beam pulse extracted from ECRIS must be chopped. The chopped beam is monitored at the observation stand consisting of $\mathrm{FC} 1$ and a profile monitor, which is located just after BM2 in LEBT as shown in Fig. 1.

The organization of this paper is as follows. In Secs. II and III, we describe Einzel lens chopper and present its background, operating principle, performance evaluations, and analysis results. In Sec. IV, we discuss the details of the longitudinal motion of the chopped beam on the basis of analysis performed by computer simulations, and compare the simulation results with experimental results. The conclusion of this paper is given in Sec. V.

\section{EINZEL LENS CHOPPER}

\section{A. Background of the KEK-DA chopper}

The beam transport region may be conveniently divided into two regions, namely, a low-energy part $(\sim 10 \mathrm{kV})$ and a high-energy part $(\sim 200 \mathrm{kV})$. If a beam chopper is installed downstream from the post-acceleration column, it must handle a beam with a voltage of $200 \mathrm{kV}$ [7], while a chopper placed upstream post-acceleration can be operated with a lower voltage of $\sim 10 \mathrm{kV}$. For each part, choppers are categorized into two types: transverse and longitudinal.

A high-voltage pulse generator with a fast response time is required in the high-energy part. The typical voltages are $200 \mathrm{kV}$ for longitudinal choppers and $20 \mathrm{kV}$ for transverse choppers. In general, both types of choppers are expensive and difficult to fabricate. In addition, $\mathrm{x}$ rays are generated as a result of collisions between the high-energy beam and matter, which demands radiation shielding. Therefore, these types of choppers have been excluded from consideration.

In the low-energy part, a transverse chopper deflects a beam transversely with the electric field induced by an RF generator, in certain cases with the aid of magnetic fields. This type of chopper has also been eliminated from our consideration, because it requires an addition chopper head (flat electrodes) and space for installation.

The extraction electrode and Einzel lens are two possible candidates for a longitudinal beam chopper in the low-energy part. Their installation locations are indicated with red circles in Fig. 4. The idea of using the extraction electrode as a chopper is similar to the ion bunching technique proposed by Jeong and collaborators, who demonstrated ion bunching by introducing an additional bunching electrode between the orifice and the extraction electrode [8]. However, the idea of inserting a new electrode was discarded due to insufficient space.

Both types of choppers require fast rise and fall times, typically of the order of nanoseconds. Such a fast response cannot be expected on the extraction electrode due to the fact that the total capacitance of the extraction electrode was $99 \mathrm{nF}$, which has been actually measured and necessarily includes all components of the ECRIS, such as ECRIS itself, large evacuation port, waveguide, and metal base plate. On the other hand, the entire assembly of Einzel lens with a small measured capacitance of $90 \mathrm{pF}$ can be expected to provide a sufficiently fast response. Consequently, an Einzel lens chopper should be applicable to the KEK digital accelerator [7].

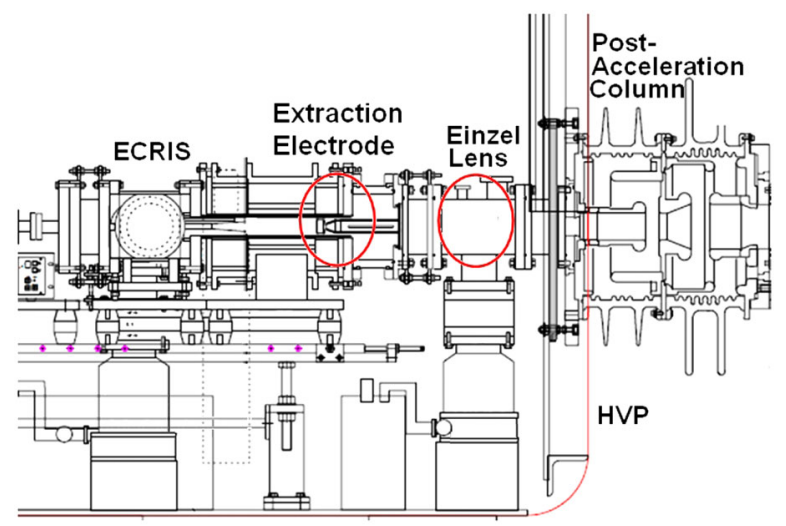

FIG. 4. Possible candidates for a longitudinal chopper. 


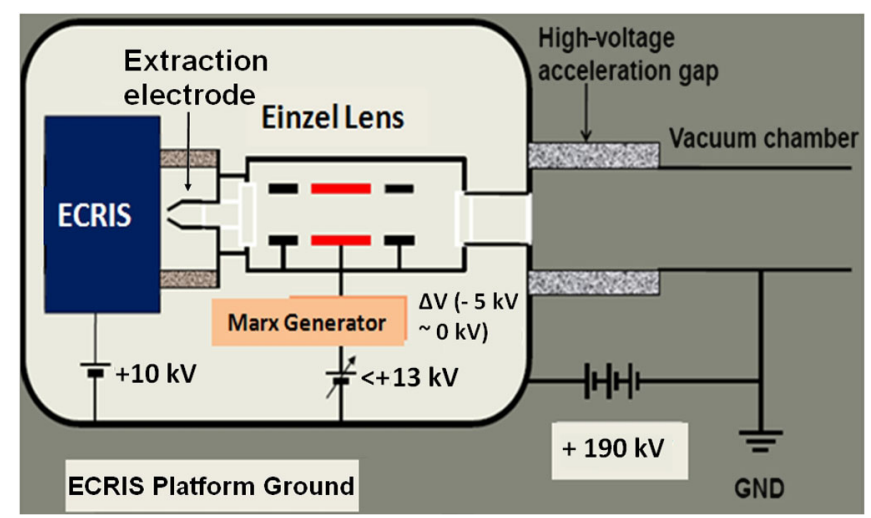

FIG. 5. Schematic view of ECRIS with the extraction system, the Einzel lens and the post-acceleration column on HVP with a voltage of $190 \mathrm{kV}$.

\section{B. Principle of the Einzel lens chopper}

An Einzel lens operates as a focusing device in transverse direction. In KEK-DA, the Einzel lens is installed immediately after ECRIS to focus the extracted beam by supplying a voltage to the middle electrode of the Einzel lens. Figure 5 schematically shows the position and electric potential of ECRIS, the Einzel lens, and the postacceleration column.

If the voltage of the middle electrode is maintained at $V_{0}$ higher than the extraction voltage of ECRIS, ions are unable to propagate downstream because of the retarding field from the electrode gap. Thus, if a rectangular negative-voltage pulse $\Delta V$ (so that $V_{0}+\Delta V<V_{0}$ ) is superposed onto the voltage of the middle electrode for a time duration $\tau$, the voltage of the middle electrode is reduced, allowing ions to propagate beyond the Einzel lens for the duration of $\tau$. This is the essential mechanism of the Einzel lens chopper, which in effect is "longitudinal gating." This effect can be realized by introducing any negative-voltage pulse power supply with fast rise and fall characteristics. A solid-state Marx generator [9] was developed for this purpose, and we refer to this type of beam chopper as an Einzel lens chopper [10], or Einzel lens longitudinal chopper in the first demonstration [9]. It bears emphasis that the present beam chopper serves both functions of transverse focusing and longitudinal chopping.

\section{Simulation and experimental results for the Marx generator}

The circuit performance of our Marx generator is simulated by using the LT Spice circuit analysis software. The schematic of the equivalent circuit of our system is shown in Fig. 6. The V1 (negative voltage) is fed into capacitors (C1 to $\mathrm{C} 4$ ) connected in parallel when the switches ( $\mathrm{S} 1$ to S4) are turned off. While the switches are maintained in the "on" position by a pulse source (V2) for a certain interval of time, the capacitors are connected in series. Then, the voltages of the four capacitors are summed and supplied to the Einzel lens. Within this interval, the beam propagates through the Einzel lens since the blocking voltage $(\mathrm{V} 3=13 \mathrm{kV})$ is reduced by the output voltage of the Marx generator $(-5 \mathrm{kV})$. Comparisons of the voltage waveforms simulated with LT Spice, and the measured waveforms for pulse durations of 3 and $5 \mu \mathrm{s}$, are shown in Fig. 7. It appears that the measured voltage waveform is consistent with the simulated one.

\section{IGUN simulation and longitudinal gating experiment}

The IGUN simulation software [11] is used to simulate the beam orbits from the ECRIS exit to the postacceleration column through the Einzel lens [6].

The beam behavior between the post-acceleration column and the Faraday cup (Fig. 1), where the IGUN software is not applicable as it assumes axial symmetry, is calculated with the transfer matrix method, and the simulation is performed for different values of the Einzel lens voltage $\left(V_{1}\right)$. Figure 8 shows the dependence of the beam current arriving at the Faraday cup as a function of the Einzel lens voltage. From this plot the optimal voltage for focusing the He ions is found to be $\sim 8 \mathrm{kV}$.

The beam trajectories of $\mathrm{He}^{1+}$ ions up to the end of the Einzel lens are shown in Fig. 9 for five values of $V_{1}$, namely, 5, 6, 8, 12, and $13 \mathrm{kV}$. At lower voltages ( 5 and $6 \mathrm{kV}$ ), the beam is underfocused, and a fraction of

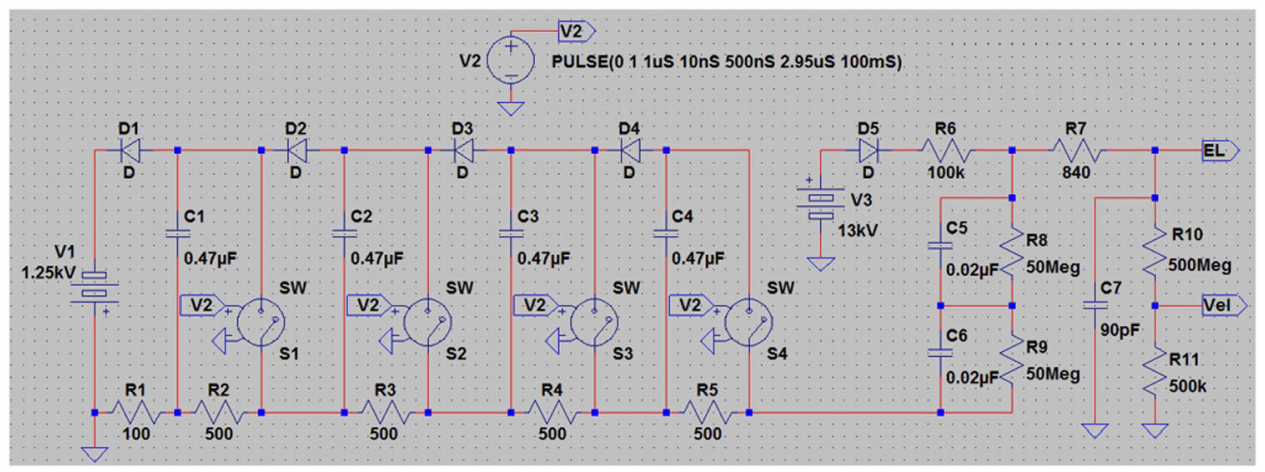

FIG. 6. Equivalent circuit of the Marx generator. 

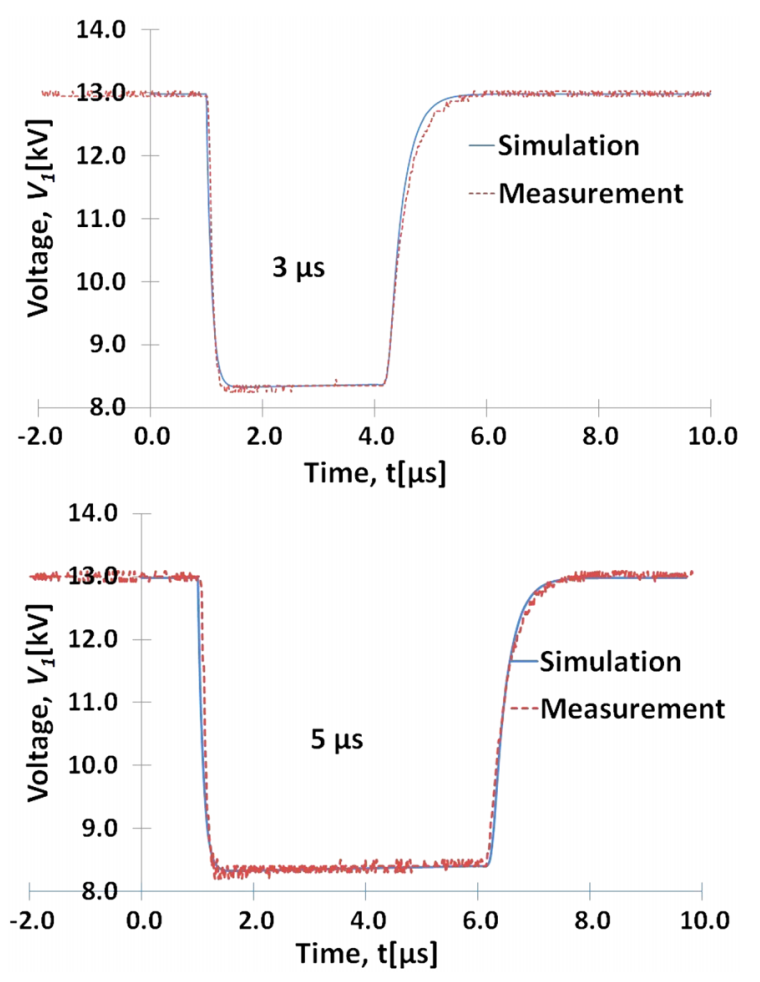

FIG. 7. Comparison of the simulated and measured voltage waveforms for pulse durations of 3 and $5 \mu \mathrm{s}$.

the beam can still propagate through the Einzel lens even at $V_{1}=12 \mathrm{kV}$. At $V_{1}=13 \mathrm{kV}$, all ions are reflected upstream and never propagate downstream. For blocking an accelerated beam of extraction voltage $10 \mathrm{kV}$, the voltage higher than the extraction voltage is required, for example $\sim 13 \mathrm{kV}$ in our system [7]. This is simply because the on-axis potential is $10 \mathrm{kV}$ with a voltage of $13 \mathrm{kV}$ on the Einzel lens electrode. The simulations as shown in Fig. 9 suggest that most of the $5 \mathrm{msec}$ beam loss inside the extraction electrode and a beam fraction of a few $\mathrm{keV}$ is lost at the surface of a chopper electrode in a very short transient time period.

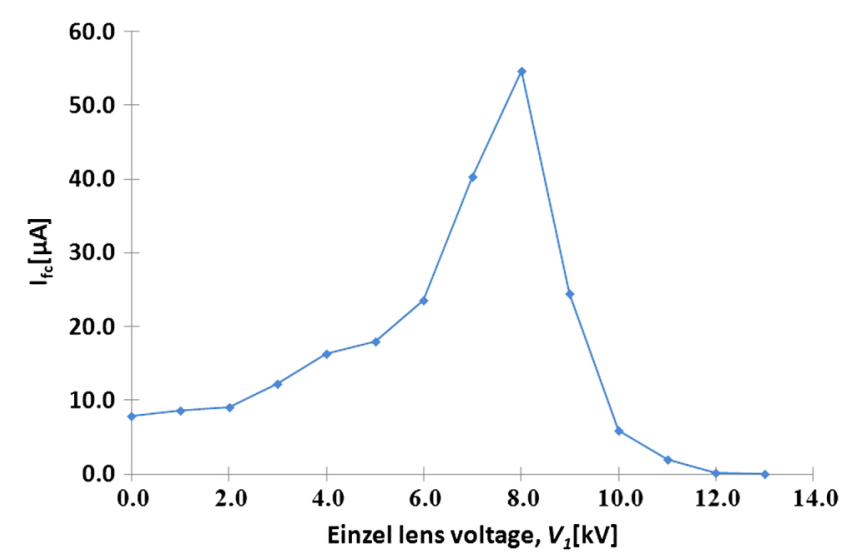

FIG. 8. Dependence of the $\mathrm{He}^{1+}$ beam currents on $V_{1}$ as simulated with IGUN and the transfer matrix method.
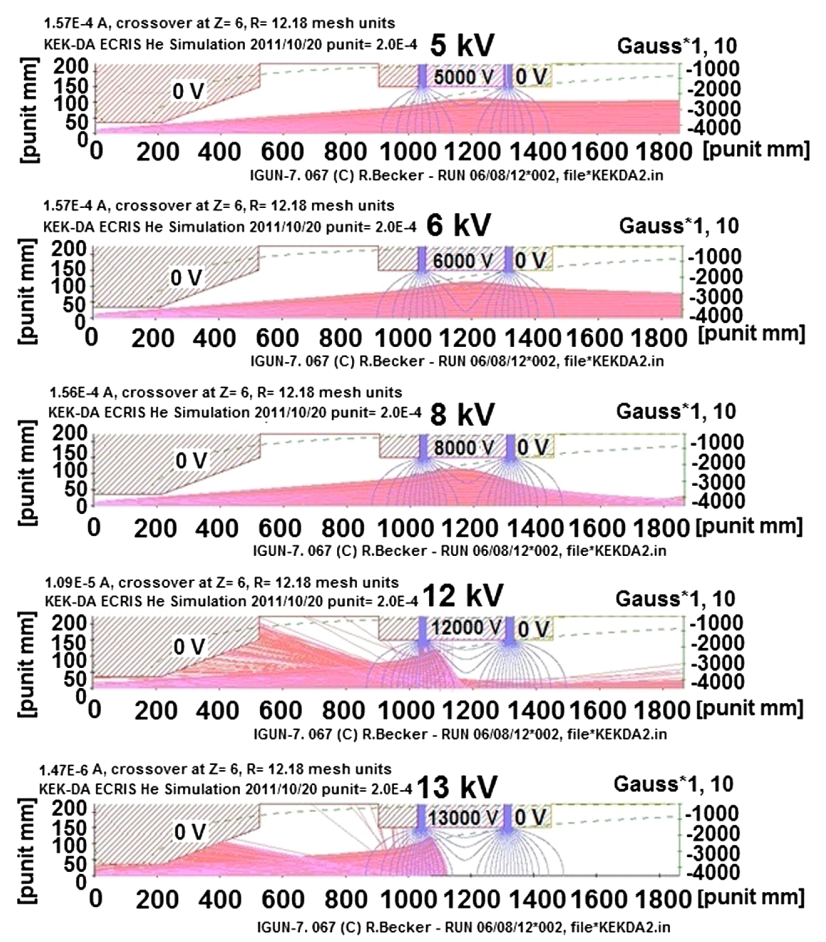

FIG. 9. IGUN simulation results for $\mathrm{He}^{1+}$ ions for the region between ECRIS exit and the end of the Einzel lens, where $V_{0}=10 \mathrm{kV}$ and $V_{1}=5,6,8,12$, and $13 \mathrm{kV}$.

To confirm that ion beam blocking is successful, the beam current of $\mathrm{He}^{1+}$ was measured with the Faraday cup, that is, $\mathrm{FCl}$ as a function of the Einzel lens voltage $\left(V_{1}\right)$. The result is shown in Fig. 10.

The result shows a sharp peak at $V_{1}=8 \mathrm{kV}$ and the beam current is drastically lower on both sides of the peak. This behavior can be qualitatively explained by taking into account the simulation results. Below $V_{1}=8 \mathrm{kV}$, the Einzel lens voltage is insufficient for focusing the beam. As a result, the beam size exceeds the effective area of the Faraday cup, and a fraction of the outer region of the propagating beam may be lost during transportation.

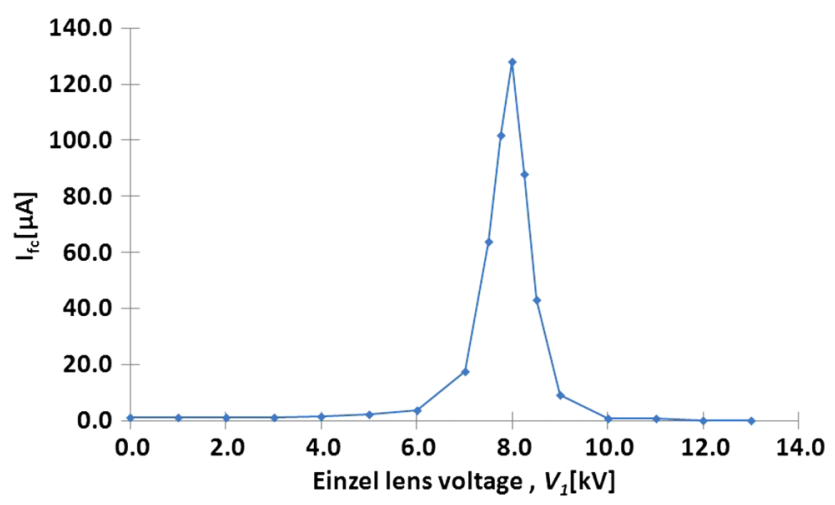

FIG. 10. Dependence of the beam current of $\mathrm{He}^{1+}$ ions on $V_{1}$ as measured with a Faraday cup. 
Above $8 \mathrm{kV}$, the beam is blown up due to overfocusing, and eventually the beam is completely blocked when the voltage exceeds the blocking voltage, which is calculated to be $13 \mathrm{kV}$. The experimental results indicate that the optimum focusing voltage applied to the middle electrode of the Einzel lens in the present configuration is $8 \mathrm{kV}$, where the extraction voltage is $10 \mathrm{kV}$ and the post-acceleration column voltage is $190 \mathrm{kV}$. Simulation and experimental results are consistent with each other with respect to the blocking and optimum focusing voltages. On the other hand, the simulation result is notably different from the experimental result in the lower region of $V_{1}$, where the spectrum is rather broad. This discrepancy may be attributed to lack of information about the transverse aperture and optics parameters, which are substantially affected by unknown remnant fields of the low-current excited guiding magnets. Here it is emphasized that the blocking voltage and matching voltage have been well reproduced in the simulation.

\section{CHOPPING EXPERIMENT}

To fully demonstrate the chopping performance of the Einzel lens chopper, the chopped beam was measured with the Faraday cup placed in the monitoring bench [9]. In the experiment, the extraction voltage of ECRIS and the static Einzel lens voltage ensuring complete blockage of beam propagation were fixed at $10 \mathrm{kV}$ and $13 \mathrm{kV}$, respectively. Since the optimized Einzel lens voltage for matching the beam optics upstream was $8 \mathrm{kV}$, a negative pulse voltage of $5 \mathrm{kV}$ and a duration of $5 \mu \mathrm{s}$ was superimposed onto the DC bias voltage of $13 \mathrm{kV}$ at the middle electrode of the Einzel lens.

\section{A. Chopping at different timings}

A typical beam pulse with a time duration of $5 \mathrm{~ms}$ extracted from ECRIS is shown in Fig. 11. The $\mathrm{He}^{1+}$ beam was chopped at three different temporal locations in the beam pulse indicated by the three colored arrows in

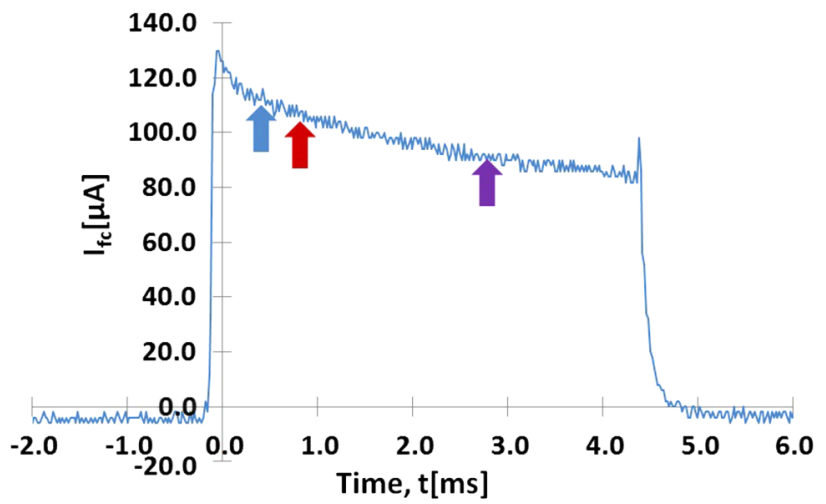

FIG. 11. $\mathrm{He}^{1+}$ ion beam pulse with a duration of $5 \mathrm{~ms}$ captured by the Faraday cup.

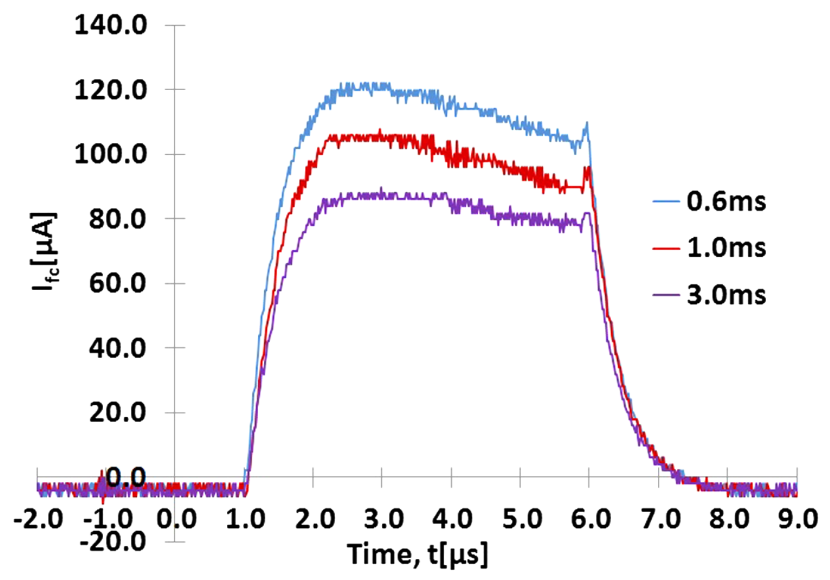

FIG. 12. Profiles of $\mathrm{He}^{1+}$ chopped beam pulses with a duration of $5 \mu \mathrm{s}$, where chopping was performed at $0.6,1.0$, and $3.0 \mathrm{~ms}$ from the pulse head of the unchopped beam.

the figure. As shown in Fig. 12, the timing of chopping was well controlled.

\section{B. Chopping with different pulse durations}

In the second experiment, the relationship between pulse profile and chopping width was investigated. The beam

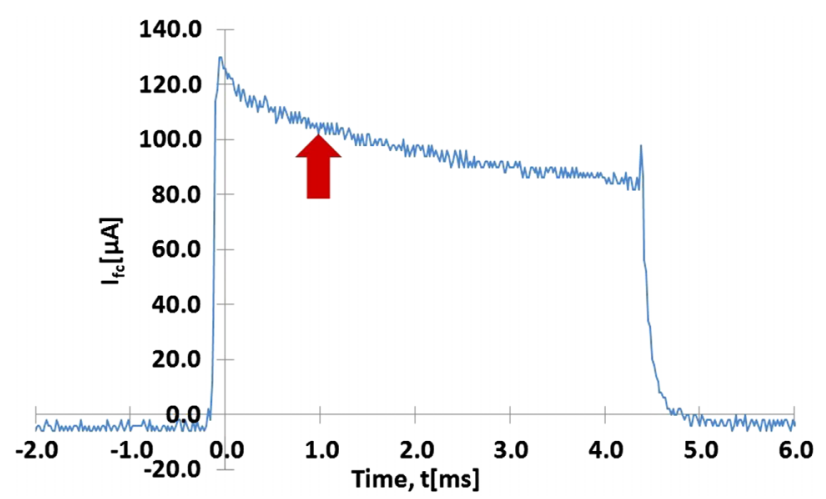

FIG. 13. $\mathrm{He}^{1+}$ ion beam pulse with a duration of $5 \mathrm{~ms}$ captured by the Faraday cup (chopped at $1 \mathrm{~ms}$ ).

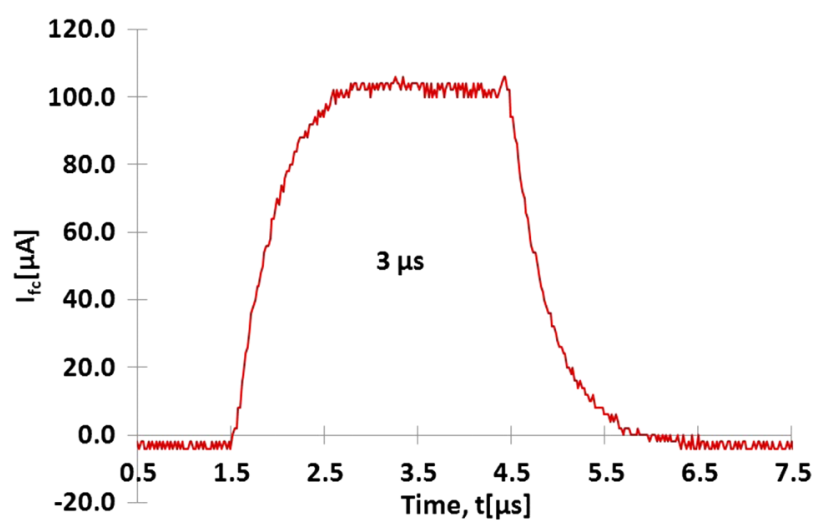

FIG. 14. Chopped beam pulse with a duration of $3 \mu \mathrm{s}$. 


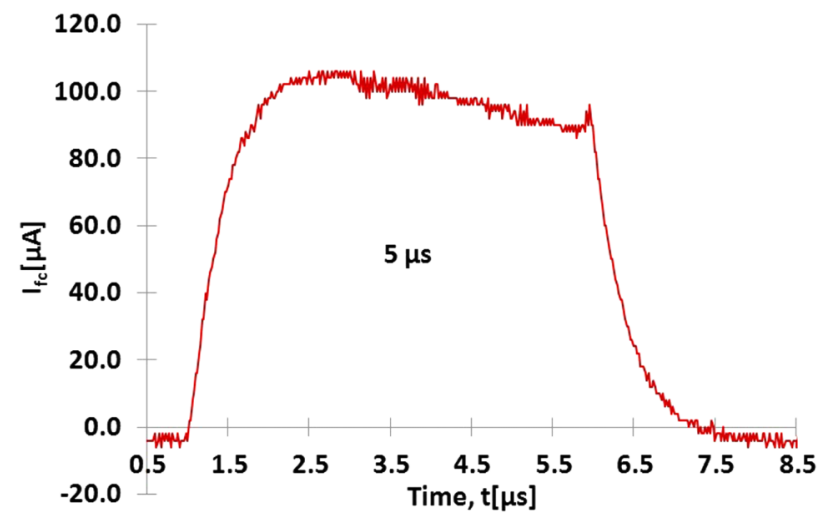

FIG. 15. Chopped beam pulse with a duration of $5 \mu \mathrm{s}$.

was chopped by setting the chopping timing at $1 \mathrm{~ms}$ with widths of 3 and $5 \mu \mathrm{s}$, as shown in Fig. 13. As shown in Figs. 14 and 15, the chopped beam width was well controlled.

\section{Reconstruction of the beam profile from Faraday cup signals}

Through the beam chopping experiments outlined above, we successfully demonstrated the performance of the Einzel lens chopper. However, it is noted that the beam signal observed with the Faraday cup does not necessarily reflect the actual beam profile in time.

Here, it is assumed that the actual beam profile and the voltage waveform applied to the Einzel lens electrode should be coincided. To address this problem in a systematic manner, we attempted to reconstruct a realistic beam profile from the measured beam signal by taking into account the response characteristics of the Faraday cup. The flow chart of the reconstruction procedure is shown in Fig. 16.

By solving the equations for the response model of a Faraday cup as described in Ref. [7], the beam current observed with the Faraday cup $\left(i_{R}\right)$ can be expressed as

$$
i_{R}=\left\{\int_{0}^{t} \frac{i_{b}}{R C}\left(e^{t^{\prime} / R C}\right) d t^{\prime}\right\}\left\{e^{-t / R C}\right\}
$$

where $R=1000 \Omega$ and $C=479 \mathrm{pF}$. In order to avoid noise effects and increase the beam signal level, the resistor of $1000 \Omega$ instead of $50 \Omega$ was used in our measurement.

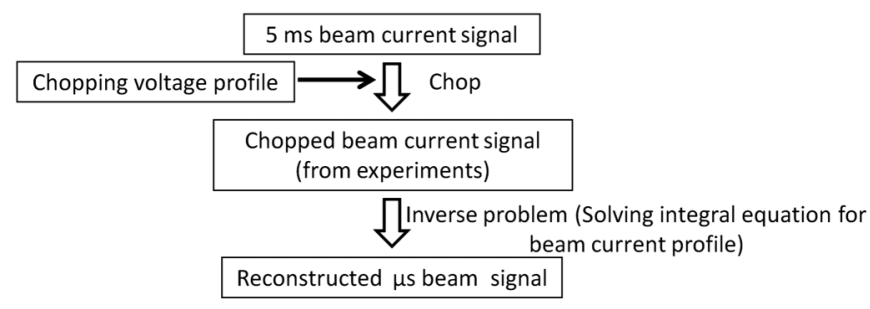

FIG. 16. Procedure of beam profile reconstruction.

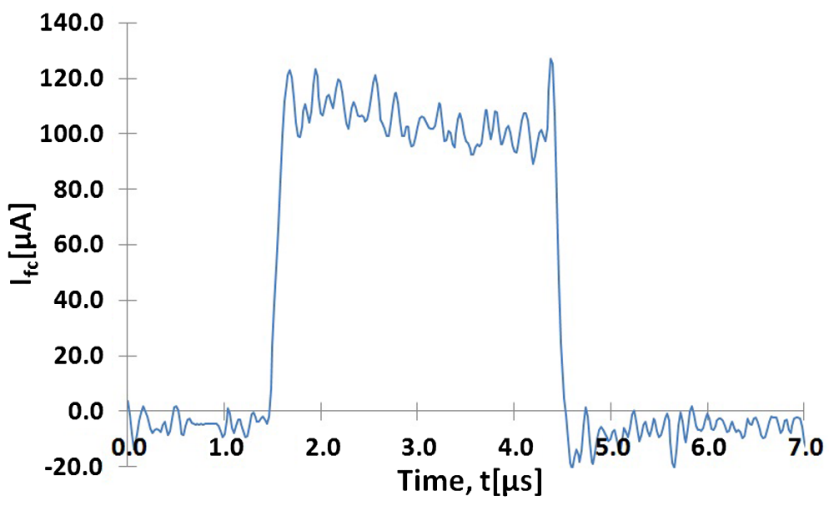

FIG. 17. Reconstructed beam profile with a duration of $3 \mu \mathrm{s}$.

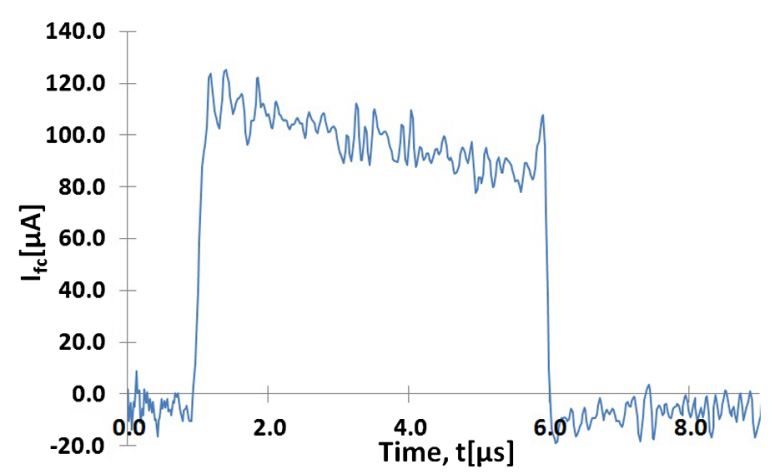

FIG. 18. Reconstructed beam profile with a duration of $5 \mu \mathrm{s}$.

The beam signal can be reconstructed by solving Eq. (1) as an inverse problem from the following equation:

$$
i_{b k}=\frac{2 R C}{\Delta t} i_{R}\left(t_{k}\right)-2 \sum_{j=1}^{k-1} i_{b k-j} e^{-j \Delta t / R C},
$$

where $i_{b 0}=0$ and $\Delta t=20 \mathrm{~ns}$. This type of reconstruction is also carried out by a method of Fourier Transformation, which has given the same result.

The reconstructed beam signals with different chopping durations are shown in Figs. 17 and 18. In all cases, a characteristic spike is seen at the end of the beam pulse profile.

\section{Comparison with chopping voltage profile}

Figure 19 shows the reconstructed beam profile with a time duration of $5 \mu \mathrm{s}$ and the chopping voltage profile applied to the Einzel lens, where the voltage profile is inverted. The reconstructed beam and the chopping voltage are not in close agreement in terms of profile shape especially in their voltage droop.

This discrepancy can be explained with the results of the blocking experiment, where the beam current captured by the Faraday cup was strongly dependent on the Einzel lens voltage. The dependence of the FC current on the chopping voltage is known, and the exact chopping voltage profile in 


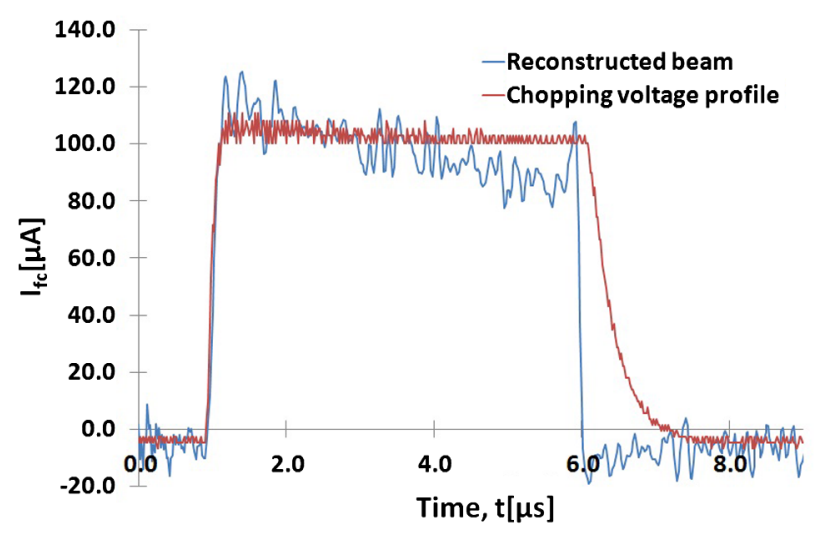

FIG. 19. Reconstructed beam pulse profile with a duration of $5 \mu \mathrm{s}$ and the chopping voltage profile in arbitrary units.

time is also known. For convenience, the observed beam current profile shown in Fig. 20 is fitted by using a Lorentzian function.

The fitting results can be summarized as follows:

$$
I_{f c}\left(V_{1}\right)=\frac{1}{V_{\text {peak }}}\left[\frac{a}{1+\left(\frac{V_{1}-b}{c}\right)^{2}}\right] .
$$

Here,

$$
\begin{aligned}
& a=1.306 \times 10^{-4}, \quad b=7.951 \times 10^{3}, \\
& c=4.029 \times 10^{2}, \quad \text { and } \quad V_{\text {peak }}=128.69 \mu \mathrm{A} .
\end{aligned}
$$

In comparing the beam profile, which is predicted by integrating Eq. (3) and the actual chopping voltage profile $V_{1}(t)=V_{1}$, with the reconstructed beam profile with a time duration of $5 \mu \mathrm{s}$, it is found that the rising and falling edges and the droop for both curves are consistent with each other as shown in Fig. 21. Hence, we conclude that the chopped beam profile almost coincides with the profile of the chopping voltage applied to the Einzel lens, with the exception of the spiky structure around the end of the pulse, which is discussed below. In other words, this fact

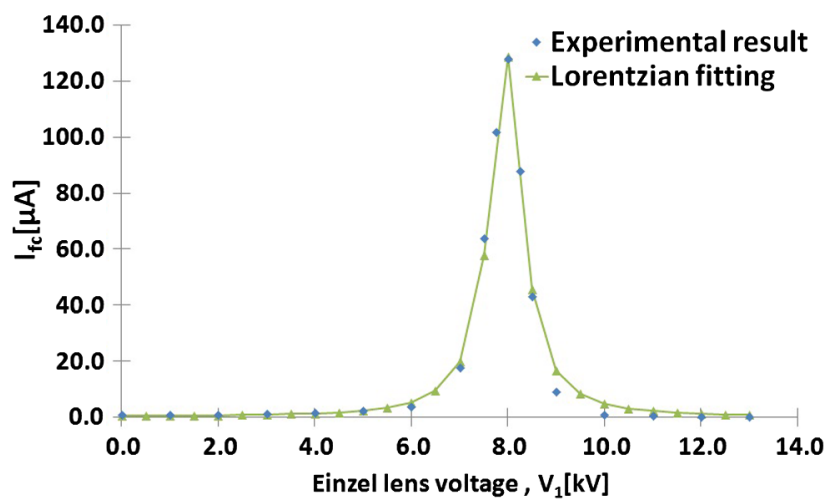

FIG. 20. Dependence of the $\mathrm{He}^{1+}$ beam current on $V_{1}$ and the corresponding fitting curve.

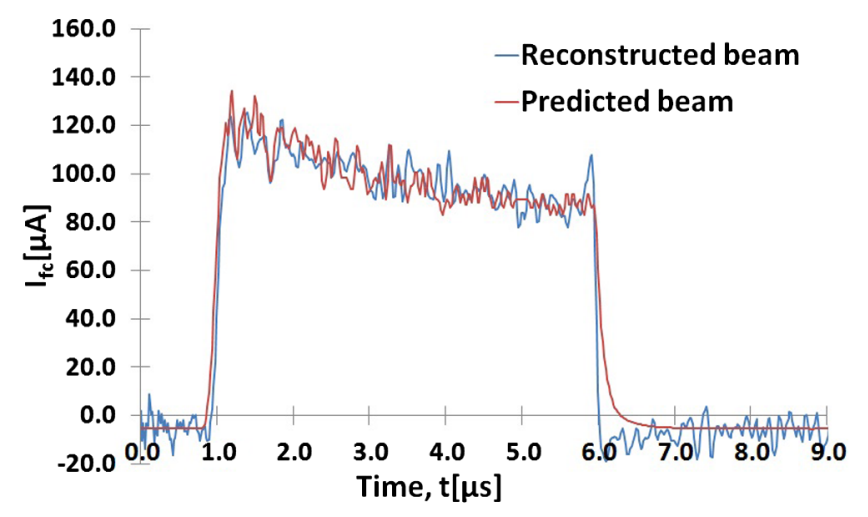

FIG. 21. Comparison of the reconstructed beam pulse with a duration of $5 \mu$ s and the predicted beam profile.

implies that beam propagation from the chopper to the monitoring bench is sensitively dominated by the transverse matching condition.

\section{SIMULATION OF THE TRANSIENT BEAM BEHAVIOR INDUCED BY AN EINZEL LENS CHOPPER}

The chopped beam is injected into the DA ring through the LEBT region. Figure 22 shows an unaccelerated circulating beam bunch signal observed with an ES bunch monitor (the yellow trace). The bunch signal shows characteristic peaks at the beginning and the end of each pulse. This may reflect some specific longitudinal beam dynamics in the chopper region. For examination of the beam behavior in the chopper region, a simple simulation program was written using Visual $* * \mathrm{C}++$ since the IGUN software cannot be used for examining particle motion in the case of timevarying electromagnetic fields.

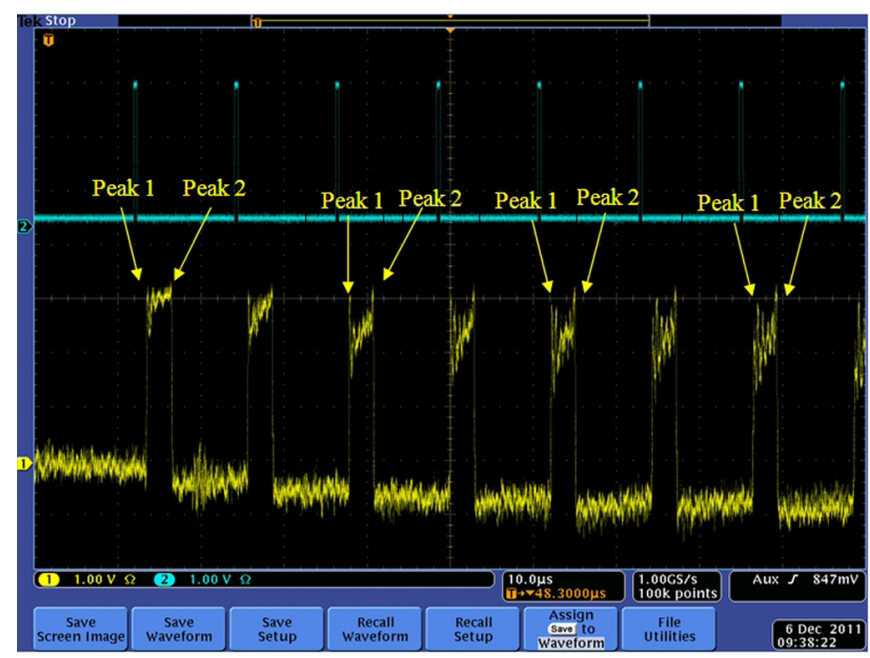

FIG. 22. Beam signal as observed with an ES bunch monitor in the DA ring. 


\section{A. Simulation procedure}

The simulation was started from an initial plane located at the extraction electrode of ECRIS and then continued through the Einzel lens, post-acceleration column, and the LEBT region, as shown in Fig. 23. The particle motion was examined under the condition of axial symmetry in the region between the initial plane and the post-acceleration column. On the other hand, axial symmetry could not be assumed for the LEBT region.

The simulation procedure is summarized in Fig. 24, and the details of the simulation and formulation are described in Ref. [7].

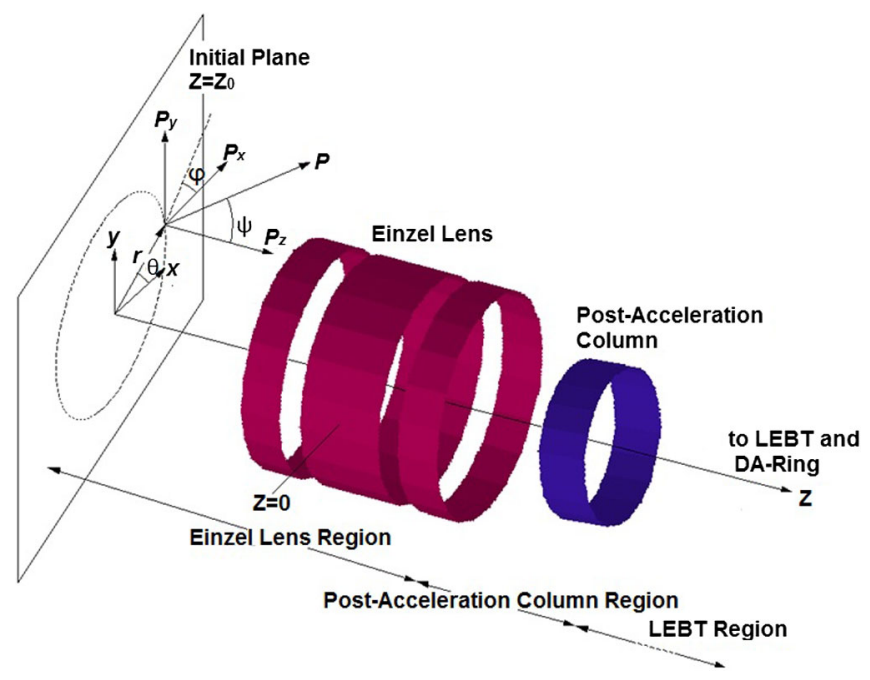

FIG. 23. Schematics of the simulation region and the coordinate used in the simulation code for the upper region from the initial plane to the end of the post-acceleration column.

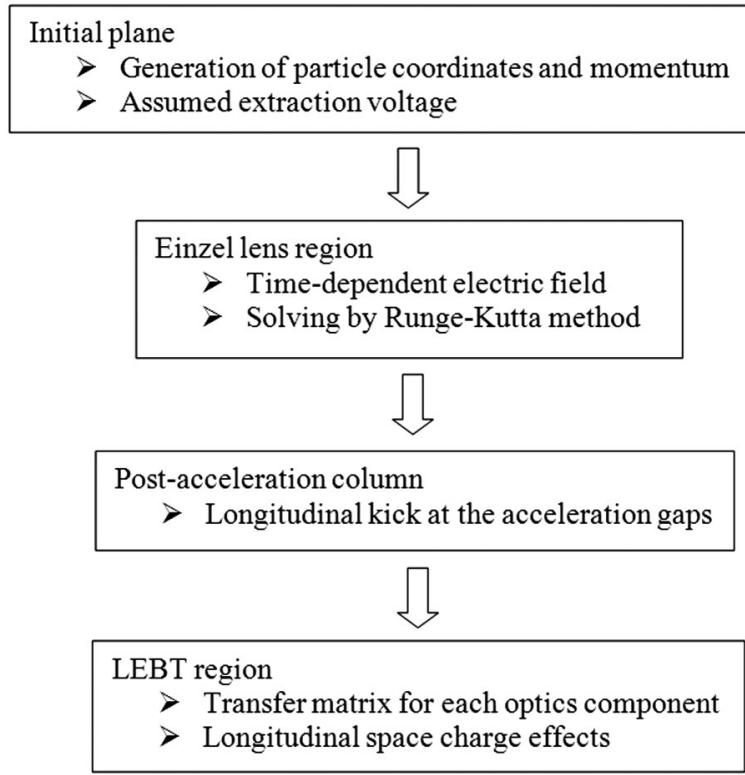

FIG. 24. Flowchart of the simulation procedure.

\section{B. Simulation results}

\section{Initial plane}

$50000 \mathrm{He}^{1+}$ macroparticles were generated at the extraction electrode of ECRIS. The transverse coordinates of each particle were generated randomly by assuming a circular beam with a radius of $3.5 \mathrm{~mm}$ and uniform density. The momentum of each particle was generated according to a Maxwell-Boltzmann distribution with a temperature of $5 \mathrm{eV}$. After momentum generation, only the $z$ component of the momentum was modified by considering an extraction voltage of $10 \mathrm{kV}$. A time between -2.0 and $5.0 \mu \mathrm{s}$ was randomly assigned to each particle. The momentum distribution is shown in Fig. 25.

\section{Einzel lens region}

The particles enter the Einzel lens region from the initial plane. According to the time-dependent voltage applied to the Einzel lens, some particles pass through the Einzel lens, and others are reflected. In this simulation, the time duration of the chopping voltage was set to $3 \mu \mathrm{s}$. Phase space distribution of particles, which pass through the Einzel lens region, is shown in Fig. 26. This result implies that momentum deviations occurred at the tail and especially at the head of the bunch.

\section{Post-acceleration region}

Throughout this region, the helium particles are accelerated with energy of $190 \mathrm{keV}$, and the longitudinal component of the momentum is boosted with this extra voltage. As a result, the large momentum deviation observed upstream is notably reduced by a factor of 20 following Eq. (4),

$$
\left(\frac{\Delta p}{p}\right)_{\mathrm{AC}}=\left(\frac{\Delta p}{p}\right)_{\mathrm{EL}}\left(\frac{V_{\mathrm{ext}}}{V_{\mathrm{tot}}}\right)
$$

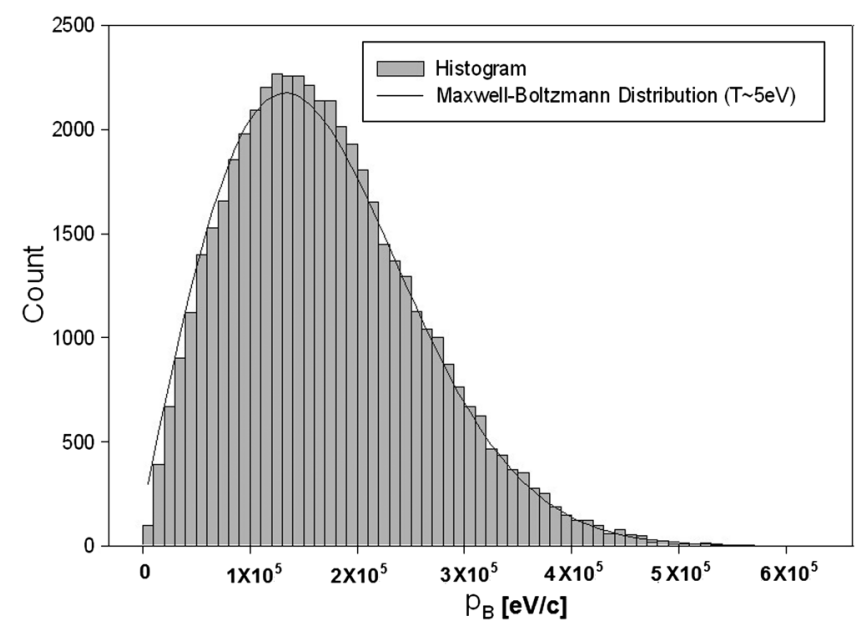

FIG. 25. Initial particle distribution and the corresponding Maxwell-Boltzmann distribution with $T=5 \mathrm{eV}$. 


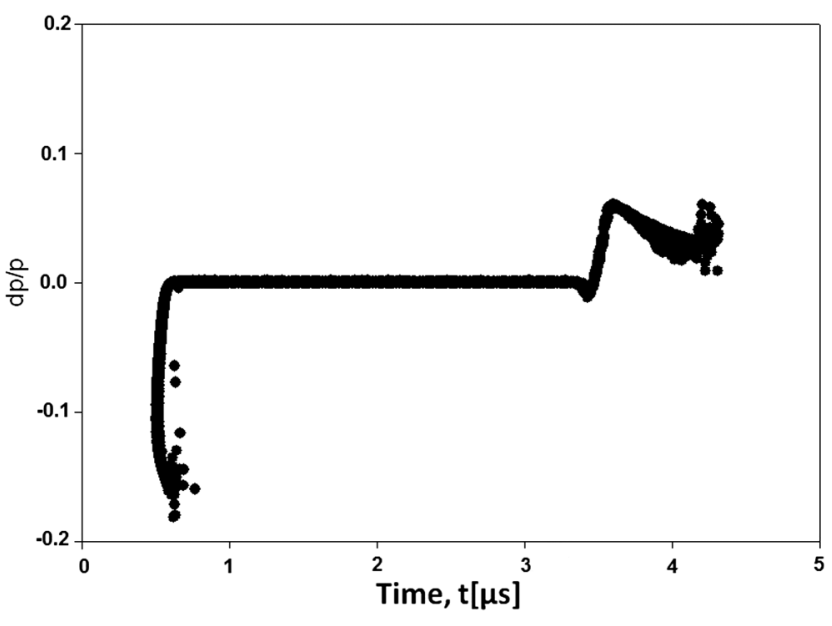

FIG. 26. Phase space distribution of particles at the end of the Einzel lens region.

Here, the indices EL and AC indicate the Einzel lens region and the post-acceleration column region, respectively. $V_{\text {ext }}$ and $V_{\text {tot }}$ denote the extraction voltage $(10 \mathrm{kV})$ and the total acceleration voltage $(200 \mathrm{kV})$, respectively.

However, Fig. 27 clearly shows that momentum deviations still exist.

\section{Drift region}

The drift region can be subdivided into two regions, namely, a short drift region for LEBT and one turn in the ring, and a long drift region in the ring.

(a) LEBT region.-The particles pass through 10 optics components, as shown in Fig. 28. The distributions of macroparticles in time at the entrance and at the end of the LEBT region are given in Fig. 29.

As shown in the figure, this simulation gives two peaks at the bunch head and tail. It is notable that the high peak of the pulse head at the entrance of LEBT disappears at the exit of LEBT. It is reasonable to consider that the pulse

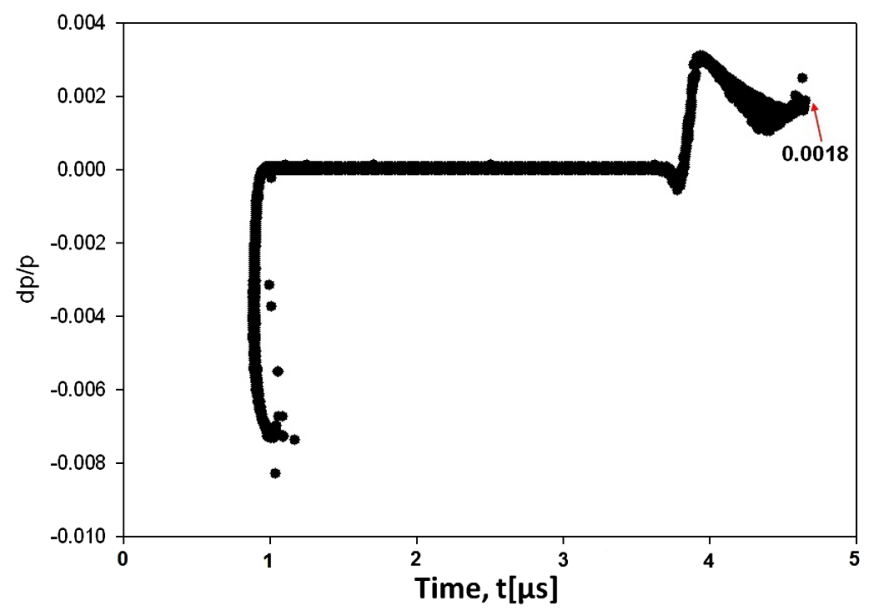

FIG. 27. Phase space distribution of particles at the end of the post-acceleration column.

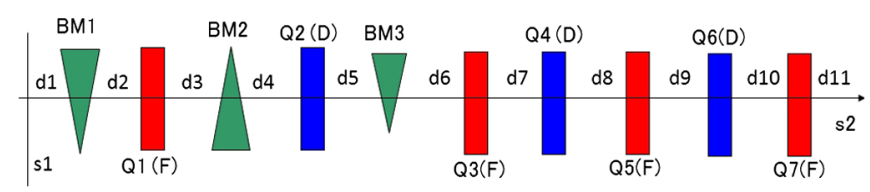

FIG. 28. Lattice components of the LEBT region (Q's are quadrupoles, D's are drift space, and BMs are bending magnets).

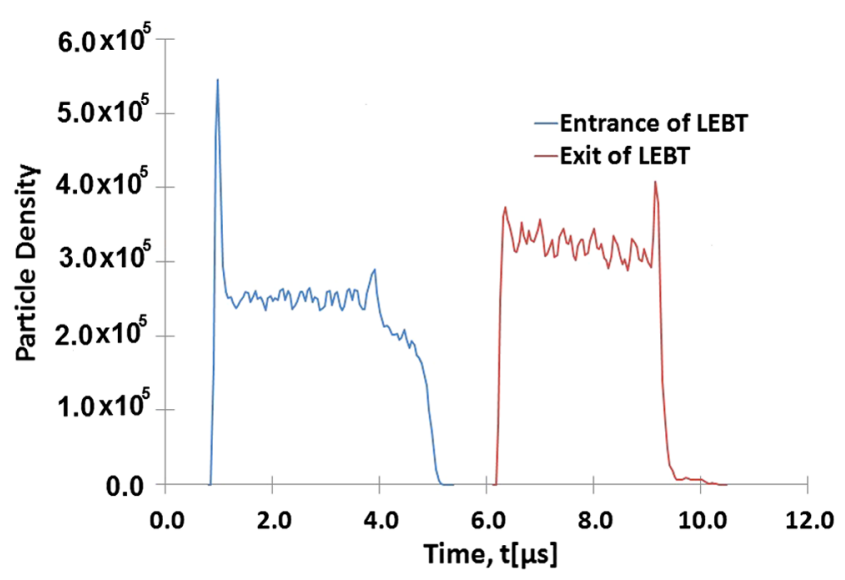

FIG. 29. Distribution of macroparticles in time at the entrance and at the end of the LEBT region.

head with relatively low momentum drifts into the pulse core.

The beam profile as acquired by the ES bunch monitor immediately after injection is plotted together with the simulation result in Fig. 30. The blue and red lines denote the simulation and the measurement results, respectively. Both results show a sharp peak at the end of the pulse.

From this result, we conclude that the major intrinsic characteristic of the Einzel lens chopper is the velocity modulation at the bunch head and tail, yielding a sharp peak at the pulse tail after a long drift. Through this simulation using macroparticles, we confirmed that the bunch head is retarded and the bunch tail moves forward

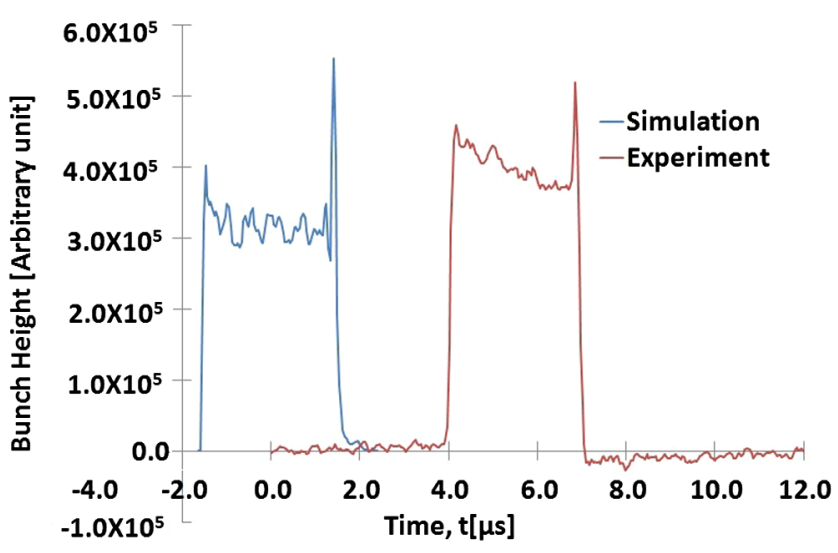

FIG. 30. Comparison of experimental and simulated bunch signals for the first turn in the ring. 
along the time axis. This motion results in the so-called "drift compression." As a result of this drift, a sharp peak is generated at the pulse tail, where a relatively large number of particles are distributed immediately after leaving the Einzel lens region, as compared with the pulse head region with a negative momentum error.

(b) Ring region.-The chopped beam is injected into the DA ring, which is known to have a sufficient momentum aperture of $\Delta p / p=1 \%$, and circulates around the ring under a constant magnetic field. The circulating beam is observed with an ES bunch monitor installed in the ring [1]. The bunch signal from the ES bunch monitor is displayed on an oscilloscope. Figure 31 shows the circulating pulse profile for $\sim 200$ turns. The obtained signals are partitioned turn by turn, and each segment is aligned vertically, forming a so-called mountain view.

Typical mountain views observed experimentally in the case of medium ion beam current $(\sim 50 \mu \mathrm{A})$ and high beam current $(\sim 100 \mu \mathrm{A})$ are plotted in Figs. 32 and 33, respectively. Strictly speaking, this is a projection of the mountain view onto the time-turn plane, where the horizontal axis represents the time within a single circulation

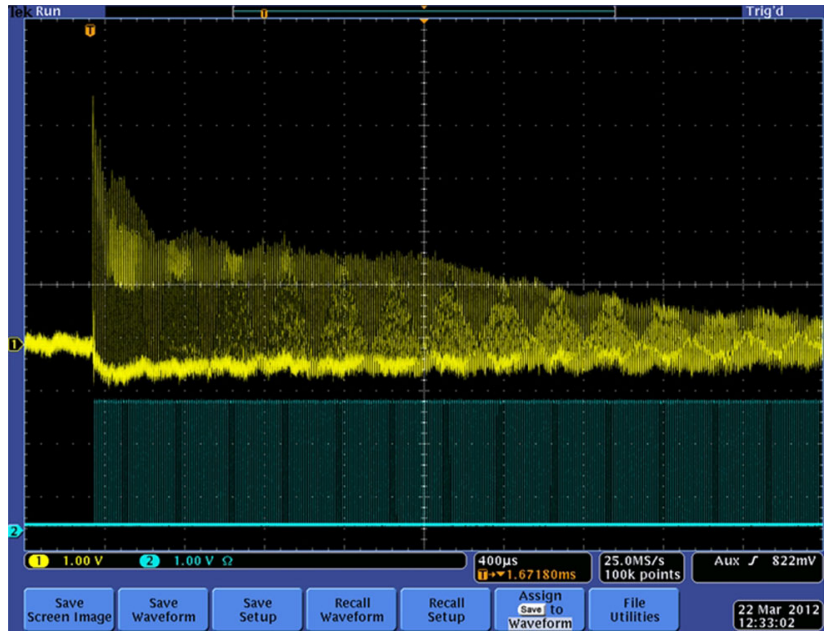

FIG. 31. Typical circulating beam signal with yellow color.

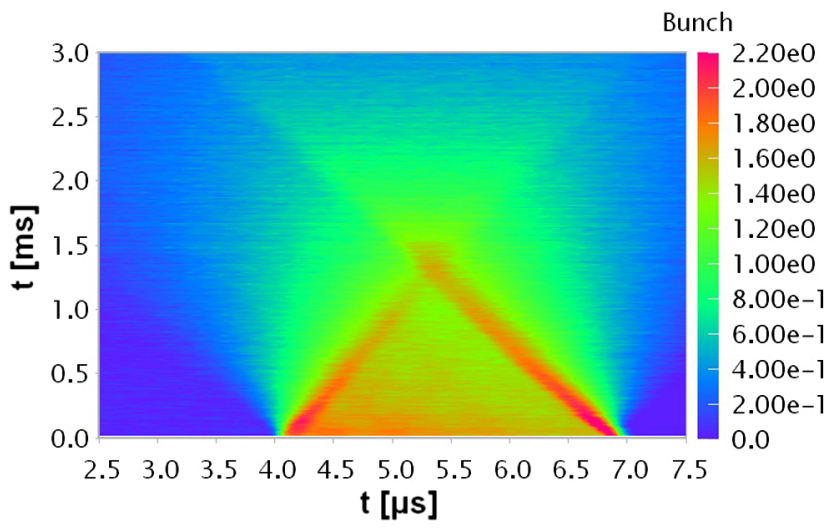

FIG. 32. Mountain view for a $\sim 50 \mu \mathrm{A}$ beam bunch signal. period, and the vertical axis is the time after injection. The simulation results are given in Figs. 34 and 35 for comparison.

In this experiment, the behavior of the chopped beam was investigated while the beam was circulating freely around the ring. We expected that a long drift may reveal some hidden characteristics of the chopped beam.

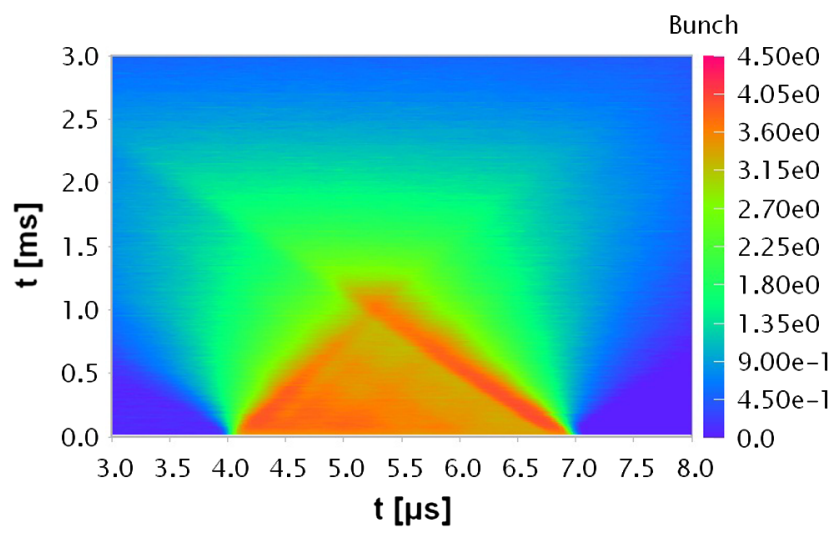

FIG. 33. Mountain view for a $100 \mu \mathrm{A}$ beam bunch signal.

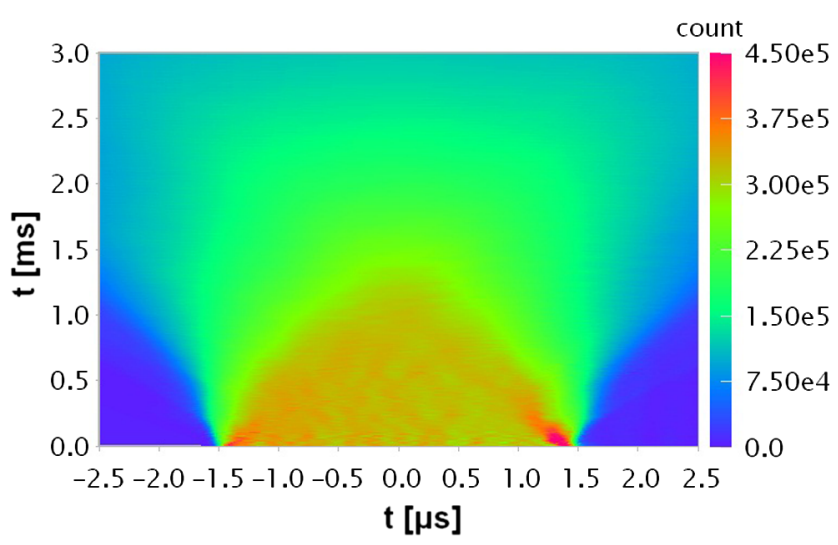

FIG. 34. Simulation result for mountain view corresponding to the $50 \mu \mathrm{A}$ beam bunch.

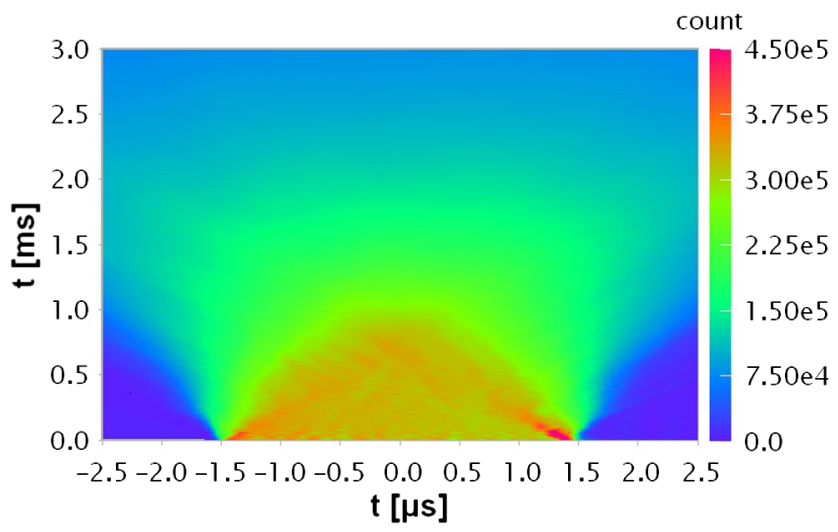

FIG. 35. Simulation result for the mountain view corresponding to a $100 \mu \mathrm{A}$ beam bunch. 
The space-charge effect was clearly present, where the diffusion of particles outside the bunch core in time depended on the beam current. This phenomenon is rather trivial. With the contribution of the longitudinal spacecharge effect, particles located at both edges of the bunch are pushed out of the bunch core. The beam crossing phenomenon was also observed. A detailed discussion is given in the next subsection.

\section{Discussion}

As mentioned in the previous subsection, the longitudinal momentum of an ion due to the transient chopper pulse voltage deviates strongly throughout the bunch, depending on the time when the particle leaves the Einzel lens region. This appears to be a common property of longitudinal choppers. Therefore, the physics of this phenomenon are described as follows.

If a static potential is applied to the Einzel lens electrode, particles should leave the Einzel lens region with their initial velocity as shown in Fig. 36. In the Einzel lens chopper discussed here, the potential changes in time as shown in Fig. 37. For convenience, the transient chopper pulse is divided into three regions with respect to time (denoted as early, intermediate, and later).

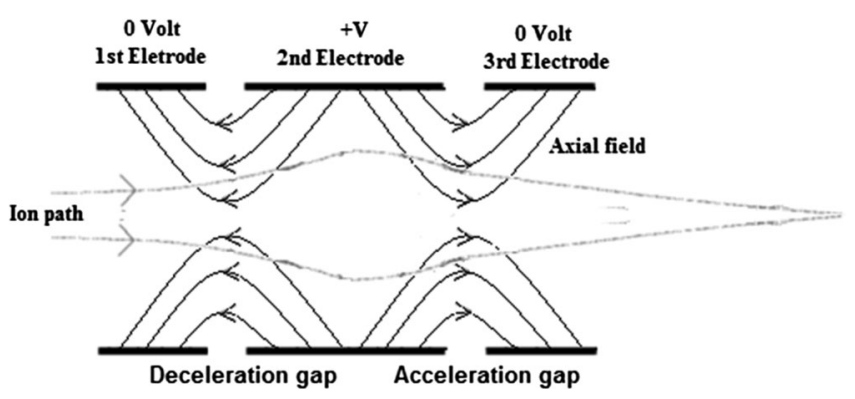

FIG. 36. Electric field lines and beam envelope in the Einzel lens region.

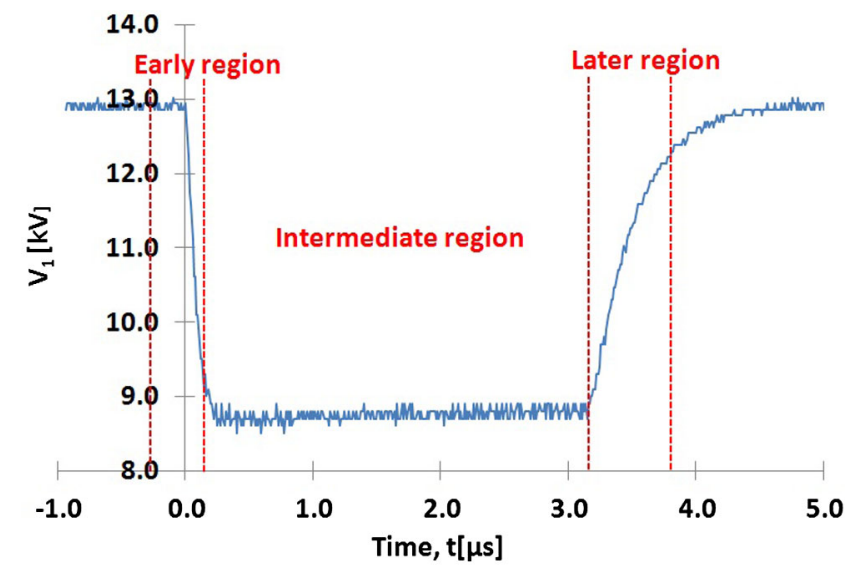

FIG. 37. Profile of the voltage applied to the middle electrode of the Einzel lens.
Assuming that a particle passes through the deceleration gap at $t_{1}$, and through the acceleration gap at $t_{2}$, during that time the particle is affected by the deceleration voltage $V\left(t_{1}\right)=V_{1}$ and the acceleration voltage $V\left(t_{2}\right)=V_{2}$. For particles passing through the deceleration gap in the early time region, $V_{1}>V_{2}$. As a result, the velocity decreases as compared with the initial velocity, and the momentum deviation becomes negative. For particles passing through the deceleration gap in the intermediate time region, $V_{1}=V_{2}$. Hence, the deceleration effect is canceled out by the acceleration effect, and the particle velocity remains unchanged. Lastly, $V_{1}<V_{2}$ for particles passing through the deceleration gap in the late time region, and as a result their velocity increases and then their momentum deviation becomes positive. In order to confirm this speculation, we conducted a simulation where particles were generated between -2.0 and $5.0 \mu \mathrm{s}$, and their trajectories in $z-v_{z}$ phase space were investigated. The simulation results are shown in Fig. 38.

In this figure, we can see a strong dependence of particle trajectories on time, and the results can be summarized as follows: (1) In the time regions of $t \leq-0.5 \mu \mathrm{s}$ and $t \geq 3.7 \mu \mathrm{s}$, particles are reflected. (2) Around $t=0 \mu \mathrm{s}$, the particle velocity decreases after passing through the Einzel lens. (3) In the time region of $0.5 \mu \mathrm{s} \leq t \leq 3.0 \mu \mathrm{s}$, the particle velocity is unchanged. (4) In the time region of $3.1 \mu \mathrm{s} \leq t \leq 3.6 \mu \mathrm{s}$, the particle velocity increases after passing through the Einzel lens.

This confirms the strong velocity modulation observed in the chopper region. This modulation survives beyond the post-acceleration column region, as shown in Fig. 27.

While a chopped beam is passing through the LEBT region, we can expect orbital evolution of the bunch profile, where the pulse head, with a negative momentum deviation, moves relatively slowly, while the pulse tail, with a positive momentum deviation, moves relatively fast, eventually resulting in a somewhat bunched pulse. The respective heights of the bunch head and tail decrease

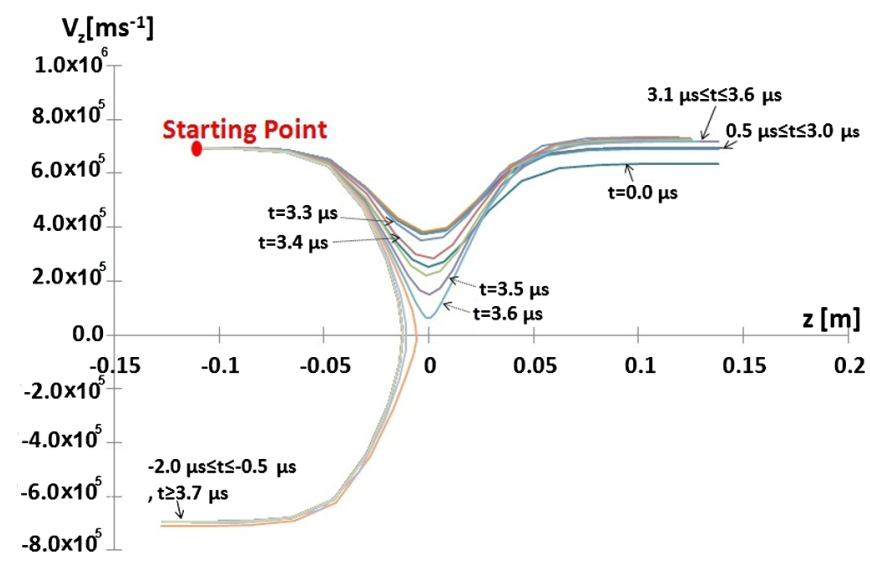

FIG. 38. Phase plot in $z-v_{z}$ space for particles injected into the Einzel lens at various times. 


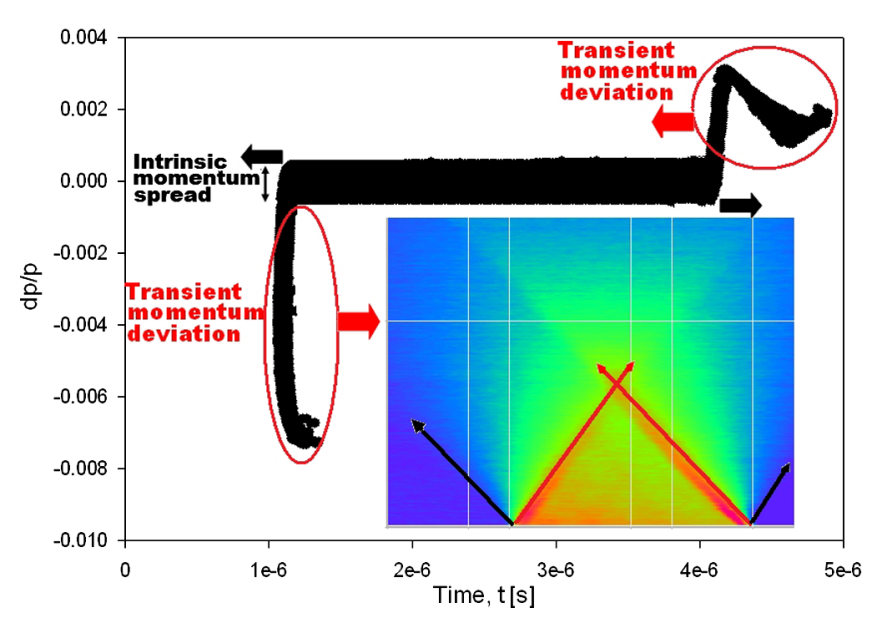

FIG. 39. Example of a mountain view with its momentum characteristics.

and increase, respectively. This is the so-called drift compression phenomenon.

Velocity modulation is present even in the ring region, which is clearly seen in Figs. 32-35, where the beam diffusion and crossing characteristics are shown. These characteristics can be explained in relation to the momentum deviation of the chopped pulse, which can be divided into two categories as shown in Fig. 39. The parts indicated with black arrows originate from the plasma formation mechanism in ECRIS and are known as "intrinsic momentum spread" for convenience. The parts indicated with red arrows originate from the "transient momentum deviation" induced by the Einzel lens chopper.

The drift of ions with a momentum error $\Delta p / p$ in phase space or in the time plane can be estimated in terms of drift time per turn as

$$
\Delta T=T \eta\left(\frac{\Delta p}{p}\right)
$$

where $T$ is the revolution period of the on-momentum particle $(\Delta p / p=0)$ and $\eta\left(=1 / \gamma_{T}^{2}-1 / \gamma^{2}\right)$ is the slippage factor of the KEK-DA ring. Since $\eta=-0.81$ for $\mathrm{He}^{1+}$ at $200 \mathrm{keV}$, the shift is to the left for particles with $\Delta p / p>0$ and to the right for particles with $\Delta p / p<0$. The momentum error is easily evaluated by substituting $\Delta T$ as obtained from the mountain views in the time-turn plane into Eq. (5). If there is nothing to disturb the momentum in the ring, the evaluated momentum error should reflect the momentum information of the injected beam as is.

Thus, it is expected that a beam with intrinsic momentum spread diffuses from the pulse head and tail as indicated with the black arrows in Fig. 39. In contrast, a beam with transient momentum deviation drifts in the opposite direction, forming a cross in the time-turn plane, as indicated in the same figure. However, this does not hold in the presence of any perturbations, such as the longitudinal space-charge effect, in which case the extent of the diffusion and the location of the crossing point may change.

The previous section presented typical experimental and simulation results for two ion beam currents. Taking into account the above speculation and the observed results, we can estimate the maximum momentum deviation. Using the following equation,

$$
\left(\frac{\Delta p}{p}\right)=\frac{\Delta T}{n T \eta}
$$

where $n$ is the number of turns after injection, we can estimate the maximum momentum deviation. For the lower half of the intrinsic momentum spread,

$$
\left(\frac{\Delta p}{p}\right)=\frac{0.23 \mu \mathrm{s}}{(0.5 \mathrm{~ms}) \times(-0.81)}=-5.7 \times 10^{-4}
$$

for the lower beam current (Fig. 32) and

$$
\left(\frac{\Delta p}{p}\right)=\frac{0.26 \mu \mathrm{s}}{(0.5 \mathrm{~ms}) \times(-0.81)}=-6.4 \times 10^{-4}
$$

for the higher beam current (Fig. 33), respectively.

Here, it should be noted that the upper half was not estimated due to the unclear diffusion in the pulse head part. The difference between beam currents can be attributed to the longitudinal space-charge effect. In fact, the simulation results, according to which

$$
\left(\frac{\Delta p}{p}\right)=\frac{0.21 \mu \mathrm{s}}{(0.5 \mathrm{~ms}) \times(-0.81)}=-5.2 \times 10^{-4}
$$

for the lower beam current (Fig. 34) and

$$
\left(\frac{\Delta p}{p}\right)=\frac{0.27 \mu \mathrm{s}}{(0.5 \mathrm{~ms}) \times(-0.81)}=-6.6 \times 10^{-4}
$$

for the higher beam current (Fig. 35), appear to strongly support this hypothesis. Nevertheless, the calculated values are somewhat different, which might be caused by the initial momentum distribution at the plasma region.

Another major concern is the transient momentum deviation induced by the Einzel lens, as shown in Fig. 26. The experimental results shown in Fig. 32 indicate the maximum momentum deviation at the tail part:

$$
\left(\frac{\Delta p}{p}\right)=\sim \frac{-0.74 \mu \mathrm{s}}{(0.5 \mathrm{~ms}) \times(-0.81)}=\sim 1.8 \times 10^{-3} .
$$

This momentum deviation is consistent with our predictions, as shown in Fig. 27.

Another notable characteristic of the mountain view is the crossing of the two portions located at the bunch head and tail immediately after injection. The timing of the crossing seems to depend on the beam current. For $50 \mu \mathrm{A}$ and $100 \mu \mathrm{A}$, the crossing occurs at $\sim 1.3 \mathrm{~ms}$ and $\sim 1.0 \mathrm{~ms}$ after injection, respectively, and these timings are reproduced fairly closely by the simulation results in both cases. This dependence of the timing of crossing on the current can also be attributed to the longitudinal 
space-charge effect. For the higher ion beam current, the longitudinal space-charge effect or the longitudinal electric field in the bunch head/tail is substantially stronger. As a result, a fraction of the particles located at the edges of the bunch accelerate or decelerate and diffuse notably faster with larger $\Delta p / p$, consistent with the simulation results shown in Figs. 34 and 35.

\section{CONCLUSION}

From the results presented in this paper, we can conclude that the satisfactory performance of the Einzel lens as a beam chopper has been successfully demonstrated by using helium ion species. To verify the performance of the chopper, the chopped beam profile was reconstructed from the Faraday cup signal, taking into account its circuit response properties. The resultant bunch profile is consistent with the beam profile as predicted from the chopping voltage. Furthermore, newly developed simulation software was used to clearly identify and demonstrate the transient effect induced in the Einzel lens chopper. It was found that the peaks in the beam profile observed downstream are mainly attributable to velocity modulation caused by the timedependent potential applied to the middle electrode of the Einzel lens. In addition, the beam motion (long drift) in the DA ring was explained well with a combination of the longitudinal space-charge effect and the above mentioned intrinsic properties of the Einzel lens chopper. However, the perturbation terms must be included into our simulation code in the future to predict the behavior of the actual beam in KEK-DA. Since the commenced operation of KEK-DA, the chopper has been used with stable and reliable performance without any failure or problem.

\section{ACKNOWLEDGMENTS}

The authors thank K. Okazaki of NAT for providing technical support in setting up ECRIS, W. Jiang of NagaokaTech for introducing the Marx generator, A. Tokuchi of JPPL for assembling the Marx generator, and T. Tanaka of NagaokaTech for assisting in beam current measurements. This work was supported by a Grant-In-Aid for Scientific Research (S) (KAKENHI No. 20224005) and a Grant-In-Aid for Scientific Research (A) (KAKENHI No. 23240082).
[1] T. Iwashita, T. Adachi, K. Takayama, K. W. Leo, T. Arai, Y. Arakida, M. Hashimoto, E. Kadokura, M. Kawai, T. Kawakubo, Tomio Kubo, K. Koyama, H. Nakanishi, K. Okazaki, K. Okamura, H. Someya, A. Takagi, A. Tokuchi, and M. Wake, Phys. Rev. ST Accel. Beams 14, 071301 (2011).

[2] K. Takayama, Y. Arakida, T. Dixit, T. Iwashita, T. Kono, E. Nakamura, K. Otsuka, Y. Shimosaki, K. Torikai, and M. Wake, Phys. Rev. Lett. 98, 054801 (2007).

[3] Induction Accelerators, edited by K. Takayama and R. Briggs (Springer, New York, 2010).

[4] K. Takayama, Y. Arakida, T. Iwashita, Y. Shimosaki, T. Dixit, and K. Torikai, J. Appl. Phys. 101, 063304 (2007); 103, 099903 (2008). Patent No. 3896420, PCT/JP2006/ 308502 (2006).

[5] K. W. Leo, T. Adachi, K. Okazaki, T. Arai, A. Tokuchi, and K. Takayama, in Proceedings of the 14th International Conference on Ion Source, Giardini-Naxos, Sicily, Italy, 2011 (ICIS, Giardini-Naxos, Sicily, Italy, 2011), p. 271 [http://icis11.lns.infn.it/].

[6] L. K. Wah, K. Takayama, T. Arai, K. Okazaki, A. Takagi, T. Adachi, K. Koyama, M. Wake, and Y. Arakida, in Proceedings of 19th International Workshop On Electron Cyclotron Resonance Ion Sources, Grenoble, France, 2010 (ECRIS, Grenoble, France, 2010), pp. 150-152 [http://www.JACoW.org].

[7] L. K. Wah, Ph. D thesis, The Graduate University for Advanced Studies, 2012.

[8] S. C. Jeong, M. Oyaizu, H. Kawakami, Y. Shirakabe, N. Ikeda, and T. Nomura, Nucl. Instrum. Methods Phys. Res., Sect. B 114, 154 (1996).

[9] T. Adachi, T. Arai, K. W. Leo, K. Takayama, and A. Tokuchi, Rev. Sci. Instrum. 82, 083305 (2011).

[10] C. Y. Tan, D. S. Bollinger, K. L. Duel, P. R. Karns, J. R. Lackey, W. A. Pellico, V. E. Scarpine, and R. E. Tomlin, in Proceedings of the 3rd International Particle Accelerator Conference, New Orleans, Louisiana, USA, 2012 (IEEE, Piscataway, NJ, 2012), pp. 3886-3888. The term "Einzel lens chopper" is used in this work. Whereas the Fermi National Accelerator Laboratory (FNAL) chopper uses a combination of Einzel lenslike electrodes as a longitudinal gating chopper, it is not applicable to transverse focusing. In this sense, the FNAL chopper is conceptually different from the chopper as described in this paper [http:// www.JACoW.org].

[11] R. Becker and W. B. Herrmannsfeldt, Rev. Sci. Instrum. 63, 2756 (1992). 